\title{
Rigid-flexible Coupling Simulation for a Three- chamber Hydraulic Cylinder of a Rocket Launcher
}

\author{
Tong $\mathrm{Xu}^{*}$ and Lijun Cao \\ School of Mechanical Engineering, Shijiazhuang, Hebei, China \\ ${ }^{*}$ Corresponding author
}

\begin{abstract}
The elevation-balancing machine is used to give the elevation angle of the landing parts of the rocket launcher, and the three-chamber hydraulic cylinder is the power device in the system. In order to obtain the dynamic characteristics of cylinder reasonably and scientifically, considering the flexible characteristics of the movable piston barrel, building a 3D model of the three-chamber hydraulic cylinder through SolidWorks software, generating a modal neutral file of movable piston barrel's flexible body in ANSYS, creating a rigid-flexible coupling analysis model and simulated in ADAMS, to obtain the corresponding actuator output curves and kinetic parameters. According to the comparison of the simulation results, the lateral dynamic characteristics of rigid-flexible coupling model compared with the rigid conditions have certain changes, the rigid-flexible coupling model can more accurate response the three-chamber hydraulic cylinder's working process. It shows that the rigid-flexible coupling model established by finite element method has higher accuracy, more in line with the needs of the study.
\end{abstract}

Keywords-three-chamber hydraulic cylinder; dynamic characteristics; rigid-flexible coupling;simulation

\section{INTRODUCTION}

In the past, vehicle-borne weapons systems were often assumed to be complex mechanical systems composed of multiple rigid bodies [1]. With the continuous development of multi-body dynamics, a flexible multi-body system dynamics theory has emerged. The rocket launcher is a complex process of rapid, high-energy and strong impact during actual gunning and launching. The deformation of the key components will directly affect the initial attitude and derailment parameters of the rocket. Therefore, in the dynamic simulation calculation, the elastic deformation of the key components needs to be considered to reduce the error of multi-body dynamics simulation results and to establish a rigid-flexible coupling dynamic model that is more in line with the actual situation.

\section{MATHEMATICAL Model OF Rigid - FleXible COUPLING THREE-CHAMBER HYDRAULIC CYLINDER}

\section{A. Flexible Multibody System Equations of Motion}

Due to its strong coupling and high nonlinearity, flexible multibody systems must be solved analytically by means of numerical methods. Lagrange multipliers are usually used to establish the equations of motion of the system [2]. The differential-algebraic equations can be expressed as :

$$
\begin{aligned}
& \boldsymbol{M} \ddot{\boldsymbol{\Gamma}}+\dot{\boldsymbol{M}} \dot{\boldsymbol{\Gamma}}-\frac{1}{2}\left[\frac{\partial \boldsymbol{M}}{\partial \boldsymbol{\Gamma}} \dot{\boldsymbol{\Gamma}}\right]^{\mathrm{T}} \dot{\boldsymbol{\Gamma}}+\boldsymbol{K} \boldsymbol{\Gamma} \\
& +\boldsymbol{F}_{\mathrm{g}}+\boldsymbol{D} \dot{\boldsymbol{\Gamma}}+\left[\frac{\partial \boldsymbol{\Psi}}{\partial \boldsymbol{\Gamma}}\right]^{\mathrm{T}} \lambda=\boldsymbol{Q}
\end{aligned}
$$

Where:, $\boldsymbol{M}, \boldsymbol{\Gamma}$ is the generalized mass matrix, generalized coordinates; $\boldsymbol{K}, \boldsymbol{D}$ is the modal stiffness matrix, modal damping matrix; $\boldsymbol{\Psi}$ corresponding algebraic constraint equations; $\lambda$ corresponding Lagrange multiplier vector; $\boldsymbol{Q}$ is generalized external force.

\section{B. Rigid-flexible Coupling System Dynamics Equation}

The rigid-flexible coupling system dynamics equation is based on the dynamics of multi-rigid body system. The Lagrange multiplier is introduced to establish the rigid body and flexible body dynamics equations respectively. Finally, the two groups are combined and the corresponding constraint equations [3-4], you can establish rigid-flexible coupling kinetics equation as:

$$
\left\{\begin{array}{l}
\boldsymbol{M} \ddot{\boldsymbol{q}}+\boldsymbol{K} \boldsymbol{q}+\lambda \boldsymbol{C}_{\mathrm{q}}^{\mathrm{T}}=\boldsymbol{Q F}+\boldsymbol{Q} \boldsymbol{V} \\
\boldsymbol{C}(q, t)=0
\end{array}\right.
$$

Where: $\boldsymbol{C}_{\mathrm{q}}$ is the Jacobian matrix of the constraint equation $\boldsymbol{C}(q, t) ; \boldsymbol{\lambda}$ is the Lagrange multiplier vector, $\boldsymbol{\lambda} \boldsymbol{C}_{\mathrm{q}}^{\mathrm{T}}$ is the generalized binding force on the flexible body.

\section{STRUCTURE AND WORKING PRINCIPLE OF THREE- \\ CHAMBER HYDRAULIC CYLINDER}

As a certain type of rocket launchers with multiple emitters, so there is full load, half load and no load conditions such as changes in order to reduce the load changes in the system to achieve a smooth steady gun control, the accumulator and the hydraulic cylinder connected to balance the part Gravity and load [5], thereby improving system efficiency.

The three-chamber cylinder is composed of a fixed piston rod, an outer cylinder barrel, a moving piston barrel, an exhaust valve, a pressure ring, a seal ring and a thin tube. The fixed piston rod is integrally connected with the outer cylinder barrel. the structure shown in Figure 1. The rising chamber and the 
dropping chamber are respectively fed by the pipe joints on the fixed piston rod and the outer cylinder, and the balance chamber is connected with the accumulator oil chamber.

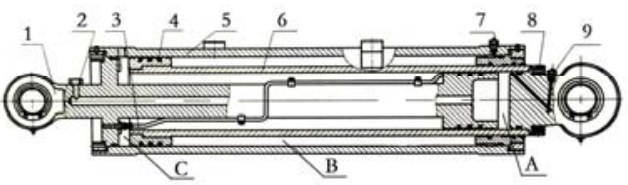

1-Fixed piston rod 2-Oil port 3-Tube 4-Seal ring 5-Outer cylinder 6-Movable piston cylinder 7-Exhaust valve 8- Pressure ring 9-Exhaust valve A-Ascending cavity B- Descent cavity C-Equilibrium cavity

FIGURE I. STRUCTURE AND WORKING PRINCIPLE OF THREECHAMBER HYDRAULIC CYLINDER

When the level machine is in the ascent state, the hydraulic oil from the tank through the gear pump, piston pump, the original pipe into the cylinder A cavity, the cylinder moves out of the piston cylinder, B cavity volume reduction will hydraulic oil into the tank, The accumulator moves to the liquid chamber under the action of the pressure of the piston in the gas chamber, and the liquid in the liquid chamber enters into the $\mathrm{C}$ chamber. Down state, the hydraulic oil into the B cavity by the pipeline, move the piston tube contraction, A cavity oil flow into the tank, and $\mathrm{C}$ cavity oil into the accumulator liquid chamber, the accumulator piston in the liquid chamber pressure to the gas Cavity direction of movement. Stopped state, the mobile cylinder stopped at a certain position, A, B, C three chamber hydraulic oil does not flow, accumulator chamber and the air chamber pressure balance, play a balance and packing effect.

\section{Modal Analysis of Three - CAVITy Moving CYLINDER BASED ON ANSYS}

Modal analysis is used to determine the vibration characteristics of mechanical components, that is, the structure's natural frequency and mode shapes, which are important parameters in structural load-bearing design. In the SolidWorks software, the kinematic model of the moving cylinder of three-chamber cylinder is established. Considering that the pressure ring and the exhaust valve have little influence on the flexibility of the components, the model is simplified. Then, the solid model was imported into the ANSYS to define the material properties. The material was 45 steel, the elastic modulus was EX $=210 \mathrm{GPa}$, the Poisson's ratio was PRXY = 0.3 and the density was DENS $=7.85 \mathrm{e} 3$. The moving cylinder was meshed by using intelligent grid. Select the flexible body and the rigid body of the external connection point to create the main node and the slave node, the establishment of rigid region of the moving piston cylinder finite element model shown in Figure 2.

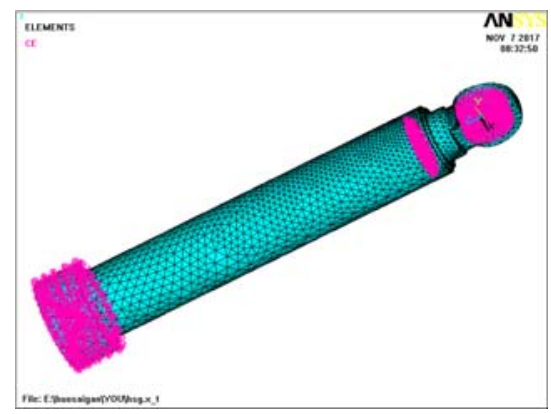

FIGURE II. FINITE ELEMENT MODEL OF THE MOVING CYLINDER

By using the modal extraction method of subspace method, the free modal analysis of the finite element model of the moving piston cylinder is made. Generally, the higher-order mode has less influence on the system [6], so only the first 12 modes are taken. Since no constraint is imposed on the moving piston cylinder, the first 6 natural frequencies are close to zero and belong to rigid mode. Natural frequency from the 7th order to take, the natural frequency of each step as shown in Table 1 , Figure 3 is 6-order non-rigid mode vibration mode cloud.
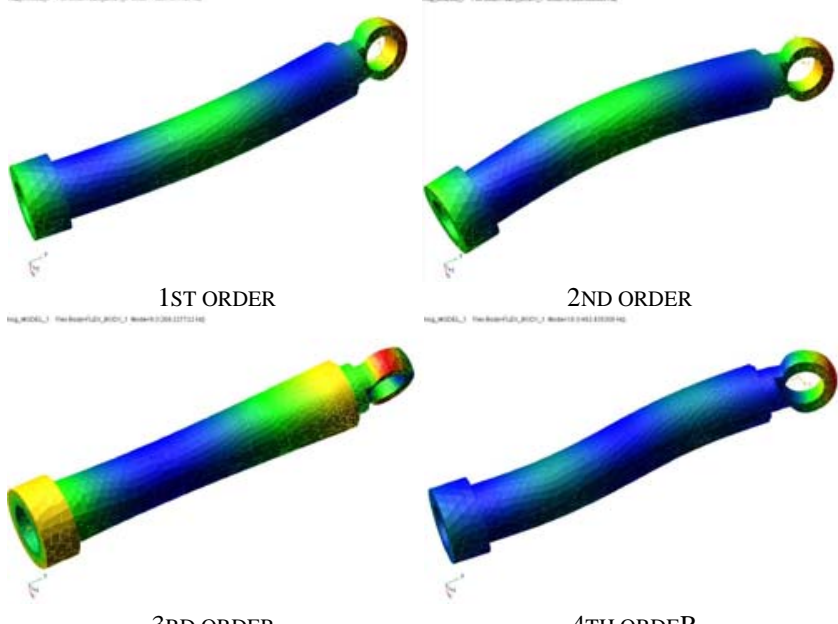

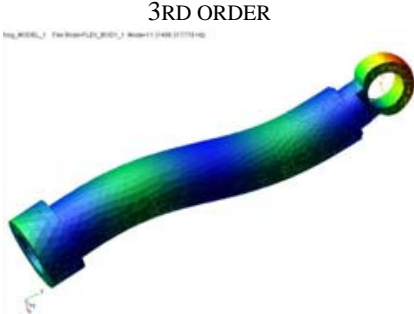

5TH ORDER

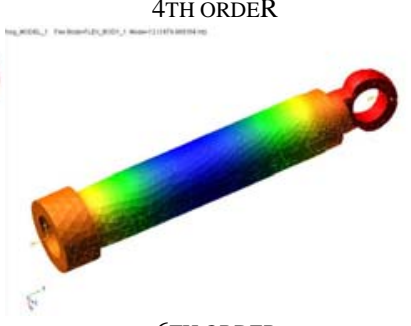

6TH ORDER
FIGURE III. MODAL VIBRATION TYPE CLOUD OF NON-RIGID

It is known from the vibration model that the first and second stages of the moving piston cylinder make lateral bending vibration, the fourth and fifth steps make longitudinal bending vibration, and the third and sixth steps are the torsional vibration of the whole body. It can be seen from this that the vibration modes of the moving piston cylinder at different natural frequencies are different. Based on the analysis results, the basic vibration modes include the overall bending and torsional modes, and the frequency range is between 600 and 
$2000 \mathrm{~Hz}$, corresponding to the non- The first six modes of rigid body. In non-rigid mode, the whole body vibrates as a whole.

\section{TABLE I. THE NATURAL FREQUENCY OF THE MOVING CYLINDER}

\begin{tabular}{|c|c|c|}
\hline $\begin{array}{c}\text { Modal } \\
\text { order }\end{array}$ & $\begin{array}{c}\text { Natural frequency } \\
/ \mathrm{Hz}\end{array}$ & $\begin{array}{c}\text { The largest relative } \\
\text { Deformation } / \mathrm{m}\end{array}$ \\
\hline 1 & 601.518 & 0.201 \\
\hline 2 & 603.008 & 0.215 \\
\hline 3 & 1269.228 & 0.163 \\
\hline 4 & 1462.435 & 0.166 \\
\hline 5 & 1486.318 & 0.210 \\
\hline 6 & 1979.989 & 0.181 \\
\hline
\end{tabular}

V. ESTABLISHMENT OF A RIGID-FLEXIBLE COUPLING MODEL FOR THREE CAVITY CYLINDERS

\section{A. The Basic Process of Rigid-flexible Coupling Analysis}

First SolidWorks 3D solid modeling and assembly, save as Parasolid (*. $\mathrm{x} \_\mathrm{t}$ ) format into ADAMS/Views, define the material properties and add constraints and movement pairs, the establishment of three-cavity rigid-body dynamics model; The processed parts are imported into ANSYS for meshing to generate modal neutral file MNF. Finally, rigid-flexible replacement is performed in ADAMS/Views [7].

\section{B. Establishing Rigid-flexible Coupling Model}

In the SolidWorks software, according to the correct coordination relationship, Gaoping machine three chamber cylinder is saved as Parasolid (*.x_t) format, and is saved to the working path of ADAMS software, then is opened in ADAMS/Views[8]. At this time, there is no constraint between the components. After deleting parts that do not affect the flexible simulation, according to the actual movement of the three cylinder, one by one, we add necessary constraints and motion pairs between the two construction.

Through the file import ANSYS macro command to generate mobile piston cylinder modal neutral file.MNF, to complete the establishment of rigid-flexible coupling model, add flexible moving cylinder after the multi-body dynamics model shown in Figure 4, Figure 5 is the degree of freedom verification for rigid-flexible coupling model.

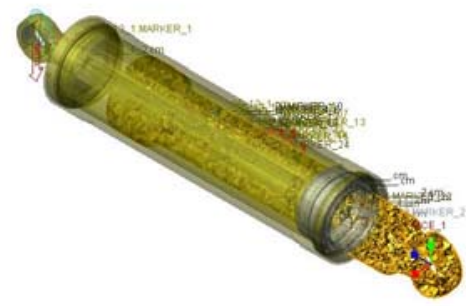

FIGURE IV. THE RIGID-FLEXIBLE COUPLING MODEL OF THREECHAMBER HYDRAULIC CYLINDER

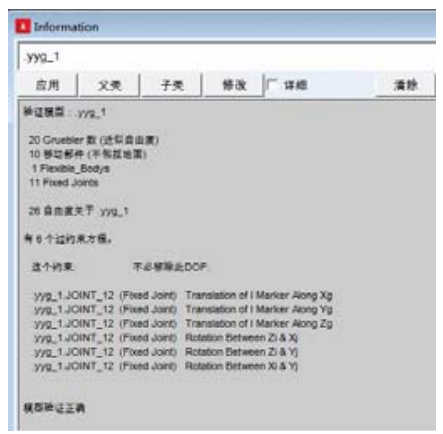

FIGURE V. THE DEGREE OF FREEDOM VERIFICATION FOR RIGIDFLEXIBLE COUPLING MODEL

\section{SimUlation AND RESUlt ANALYSIS}

The ADAMS/Solver solver was used to calculate the motion parameters of the rigid body and the rigid-flexible coupling model, and the simulation curves were obtained in the post-processing module. At the same time, the corresponding data was derived to draw the contrast curve in MATLAB. The simulation time is defined as $1 \mathrm{~s}$ and the simulation step is set as 0.01s. Fig. 6 shows the velocity curves of the piston cylinder in different directions along the axial direction of the moving piston cylinder. The dashed line indicates the rigid body dynamic model and the solid line indicates the rigid-flexible coupling dynamic model (the same below).

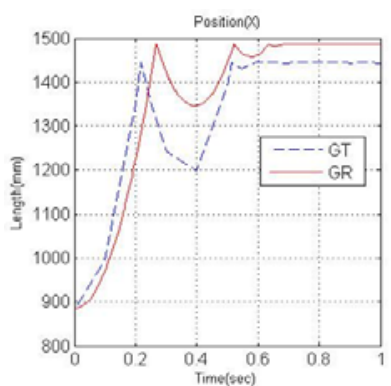

(a)

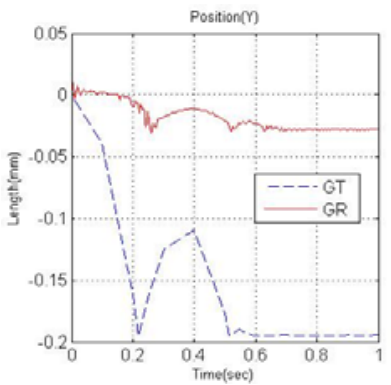

(b)

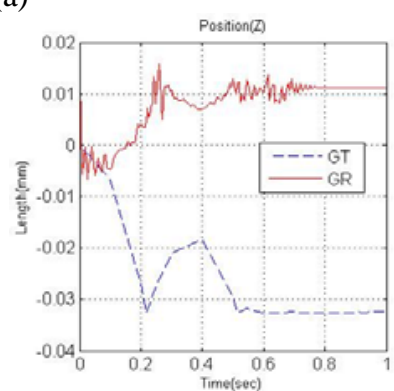

(c)
FIGURE VI. THE DISPLACEMENT CURVE OF MOVING CYLINDER IN ALL DIRECTIONS

From the comparison chart of the curve, it can be seen that the displacements of the moving cylinder in the two models are basically the same in the $\mathrm{X}$ direction, while the rigid-flexible coupling model lags slightly behind the rigid body model due to the pre-deformation of the flexible body at the beginning of the motion At the same time, due to the deformation of the flexible body, the axial displacement of the rigid-flexible 
coupling model is slightly larger than that of the rigid body model. In the $\mathrm{Y}$ and $\mathrm{Z}$ directions, the rigid body model's motion trend is consistent, the flexible body is compressed resulting in the rigid-flexible coupling model in the direction of the radial force displacement is significantly smaller than the rigid body model, and because the gravity is set to Y-axis negative direction, so the Y-axis displacement Is negative, Z-direction displacement is positive.

As can be seen from Figure 7, the velocities of the two models are basically the same in the direction of the force. The rigid-flexible coupling model has a certain speed lag due to the elastic deformation and periodically decays to zero. At the same time, the elastic deformation causes the lateral speed of the moving cylinder to increase greatly, which is consistent with the theory and the actual situation.

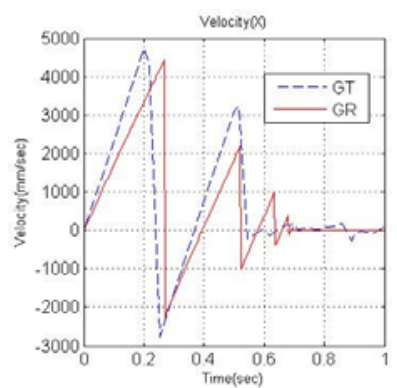

(a)

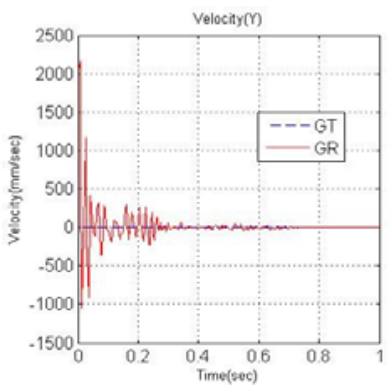

(b)

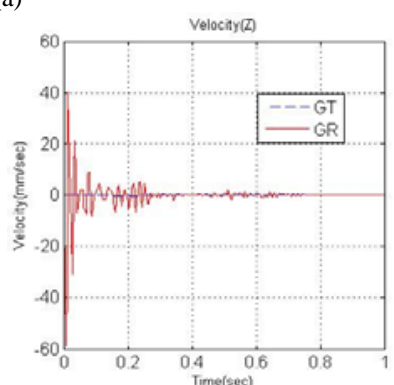

(c)
FIGURE VII. THE VELOCITY CURVE OF MOVING CYLINDER IN ALL DIRECTIONS

Select the moving piston cylinder and the guide sleeve contact surface as the reference surface, draw rigid body and rigid-flexible coupling model where the force curve shown in Figure 8. From the figure, it can be seen that the radial force of the rigid-flexible coupling model is obviously smaller than that of the rigid-body model. At the same time, due to the influence of the flexible body, the peak value of the axial force in the rigid-flexible coupling model is small and relatively lag. Thus, in the design and verification of this part, you can directly refer to the rigid body model simulation results, you can meet the actual needs.

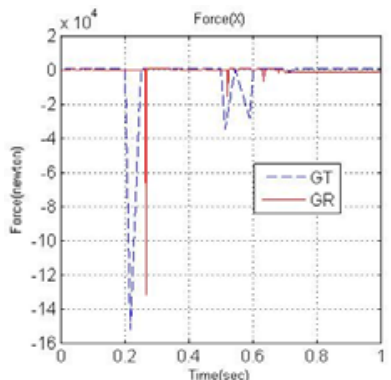

(a)

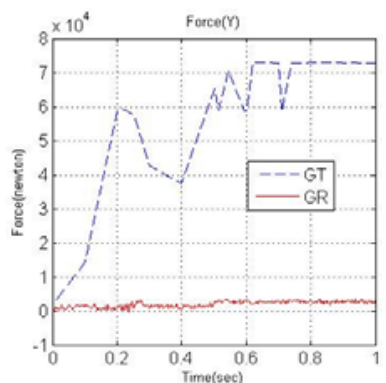

(b)

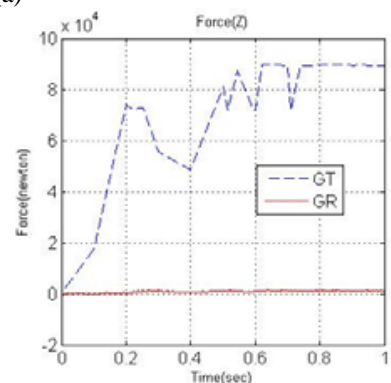

(c)
FIGURE VIII. THE CONTACT FORCE CURVE OF MOVING CYLINDER AND GUIDE SLEEVE

\section{CONCLUSION}

(1)In the SolidWorks software, a three-dimensional solid model of each component of a rocket Kao-Ping machine was built. The moving cylinder was introduced into the finite element analysis software ANSYS for modal analysis. The first 12 orders of natural frequency and total mode displacement were calculated and output Vibrational cloud. From the analysis results, it can be seen that the vibration modes of the moving piston cylinder are different at different natural frequencies, and the difference between the operating frequency and the natural frequency is large, so the resonance damage cylinder will not be produced, and the daily frequency should be kept away from the natural frequency.

(2)The ANSYS macro command was used to generate modal neutral file with the suffix of .MNF suffix. The rigidflexible coupled model of the high-plane three-cavity cylinder was established in ADAMS. Comparing with the rigid-body simulation, the rigid-flexible coupled model can better reflect the movement The kinematic and dynamic characteristics of the piston barrel in all directions lay the foundation for further simulation analysis.

\section{REFERENCES}

[1] Zhenyu Du,Xuezhi Wang,Yongqiang Cheng.Simulation Analysis of Launching Process of Vehicular Missile’s Rigid-flexible Coupling System[J]. Journal of Projectiles,Rockets,Missiles and Guidance. 2017,37(1):9-12

[2] Wenhu Huang,Chengxun Shao.Flexible Body System Dynamics[M]. Science Publishing Company,1996.

[3] Degui Liu,Jinggao Fei. Digital Simulation Algorithm for Dynamic System[M]. Science Publishing Company,2000.

[4] Liping Chen.Mechanical System Dynamics Analysis and ADAMS Application Tutorial[M]. Tsinghua University Press, 2005. 
[5] Junzheng Wang,Jiangbo Zhao,Shoukun Wang.The Development and Future Trends of Electro-hydraulic Servo Technology[J]. Chinese Hydraulics, 2014, (5): 1-12.

[6] Chengbao Yu. Modal Test and Analysis of Artillery System[D]. Nanjing University of Science and Technology,2007.

[7] Chao Wang,Xiuhua Gao.Rigid-flexible Coupling Analysis for Steering System of Multi-axle Vehicle[J]. Machinery Design, 2011, (11): 202204.

[8] Junhu Li,Weibing Wang.Dynamics Research of Double Cross Universal Joints Based on Rigid-flexible Coupling[J].Journal of Mechanical Transmission, 2016, (10): 33-37. 